\title{
Combined User and Antenna Selection in Massive MIMO Using Precoding Technique
}

\author{
Tasher Ali Sheikh, Joyatri Bora and Md. Anwar Hussain \\ Department of Electronics and Communication Engineering, North Eastern \\ Regional Institute of Science and Technology, Arunachal Pradesh, India-791109 \\ tasher372@gmail.com,bjoyatri@yahoo.com,ah@nerist.ac.in
}

\begin{abstract}
We propose here joint semi-orthogonal user selection and antenna selection algorithm based on precoding scheme. The focus of this proposed algorithm is to increase the system sumrate and decrease the complexity. We select and scheduled users from a large number of users based on semi-orthogonality condition among them. Here, we select only the maximum channel gain antennas to maximize the system sumrate. Subsequently, the user selection and antenna selection have been scheduled in an adequate manner in order to achieve the maximum system sumrate. We calculate the system sumrate for two scenarios: firstly, by considering the interference and secondly without considering the interference. We achieve maximum system sumrate at MMSE and lowest at without precoding while considering the interference. However, when we do not consider the interference we obtain lowest sumrate at MMSE and maximum at without precoding. Here, we apply the precoding scheme to increase the system sumrate and we gain $30 \%$ to $40 \%$ higher system sumrate compared to without precoding when interference is considered. Thus, we obtain higher sumrate in our proposed algorithms compared to other existing work.
\end{abstract}

Keywords: 5G; Massive MIMO; Users selection; Antenna selection; Sumrate; Interference

\section{Introduction}

The continuous increasing demand of wireless communication systems cannot meet by existing $3 \mathrm{G}$ and $4 \mathrm{G}$ technology with limited spectrum. The users to avail high data rate facility in future $5 \mathrm{G}$, it is required a high provisioned technology. To take hold of the benefits of future 5G, an emerging technology has been introduced which has most promising potential to offer high data rate to users within limited spectrum called Massive Multiple Input Multiple Output (MIMO) [1]. Massive MIMO is the fastest technology in wireless communication systems capable to offer higher data rate to the large number of users in upcoming 5G networks. As massive MIMO has large number of antennas and users, so there is a huge complexity to schedule limited number of antennas and select users from massive amount of antennas and users respectively. Moreover, one of the major advantage of massive MIMO is that there is a probability of getting more and more number of semi-orthogonal users as it has large number of users [2]. Also, simple linear encoding and decoding techniques work optimally [3]. Therefore, to implement, it needs same amount of RF chain as base station (BS) antennas. This has increased the fabrication cost, power consumption as well as system complexity [4]. To decrease computational complexity, power consumption and fabrication cost in this system, an adequate users and antenna selection, and scheduling is mostly needed. It is investigated that when some

Received (April 23, 2018), Review Result (June 28, 2018), Accepted (July 6, 2018) 
good channel conditioned antennas and users are selected from high dimensional massive MIMO, then system's capacity significantly improves. A Two-step largest MSV based (LMVS-JASUS) algorithm is proposed in [5] where in the first step SDBAB-JASUS algorithm is developed for reduction of computational complexity but does not achieve significant system sum-rate, and in second step swapping base JASUS algorithm has enhanced the system sumrate but with higher computational complexity. For antenna scheduling and user selection, brand and bound (BAB) and brute-force search (BFS) algorithms are presented in [6]. Joint antenna selection and user scheduling (JASUS) algorithm which iteratively remove those antennas and users that have least involvement to the systems are suggested in [7]. In [8] proposed three iterative algorithms for joint user scheduling and antenna selection in distributed massive MIMO systems with backhaul capacity. Among these three, the first one is norm based JASUS algorithm that lessens system complexity but does not provide significant system sumrate. The second greedy based JASUS algorithm offers maximum system sum rate but has higher computational complexity. The third TCB-JASUS algorithm balances system sumrate and computational complexity but cannot totally resolve the problem of complexity. A two stage efficient antenna selection and user-scheduling algorithm is suggested in massive MIMO-NOMA scenario [9]. In the first single-band two-users, massive MIMO NOMA situation efficient search algorithm has been proposed to select antenna by limiting the total number of antennas in a system that directly influences on system sumrate. And in the second algorithm, massive MIMO-NOMA multi-band multi-user setup combined with AU algorithm select the antennas and users whose gain is directly beneficial in total gain of a system, resulting in increase of the sumrate.

One of the major drawback of algorithms proposed in [5], [7-9], is that it only works for the small number of antennas and users in a massive MIMO based systems and cannot work for the large dimensional massive MIMO systems. For this arduous problem, we have proposed two combined scheme of simultaneously selecting semi-orthogonal users and scheduling antennas which have maximum channel gain so that it offers maximum average system sumrate. Therefore, at any instant of time, it provides maximum average system sumrate and reduces the system complexity.

The remaining part of the paper is organized as follows. The system model and problem formulation are discuss in Section 2. The two proposed algorithms are discussed in Section 3. The analysis of result is presented in Section 4 and conclusion part discussed after Section 4. The symbolic representation are as follow: $(.)^{\mathrm{H}}$ Hermitian of a matrix, \|\|$^{2}$ norm of a vector, $|\cdot|$ absolute value of a scalar, and $(y)^{+}$represents max $b(0, y)$.

\section{System Model and Problem Formulation}

\subsection{System Model}

In this system, we assume a massive MIMO system with $M$ number of BS antennas and $N$ number of users with single receive antenna. $G$ users are selected among total $N$ users, and select and schedule $G$ number of antennas from $M$ BS antennas with selected users. The received signal at $k^{\text {th }}$ user is given by equation (1)

$$
y_{k}=h_{k}^{H} x+\sigma_{k}
$$

Where $k=1, \ldots N$ and $h_{k} \in C^{M \times N}$ is the $k^{\text {th }}$ channel vector in the channel matrix. We consider that all the gains of the channel matrix is quasi-static independent and identically distributed (i.i.d.) Rayleigh fading channel, $x \in C^{N \times 1}$ transmitted signal vector from $N$ user to all BS antenna with power constraint $E=\left[\|x\|^{2}\right]=\rho$ and $\sigma_{k} \in C^{M \times 1}$ is 
the noise according to distribution of independent complex Gaussian with zero mean and unit variance. The objective of the algorithm-1 of this paper is to find out semi-orthogonal users set $U_{S O U}$ from $N$ users and store them in a matrix by columns [10]. We know that vector $Y$ and $Z$ are semi-orthogonal for orthogonality $\alpha \geq 0\left(\right.$ i.e. $\left.Y \perp_{\alpha} Z\right)$ if equation (2) hold.

$$
\alpha \geq \frac{\left|Y^{H} Z\right|}{\|Y\|\|Z\|}
$$

To obtain higher sumrate in MIMO broadcast channel, DPC are usually used but it is not possible to implement practically in massive MIMO systems [12]. Therefore, to acquire higher sumrate in massive MIMO broadcast channel we adopt here simple linear precoding techniques. With the ZF and MMSE linear precoding schemes, we obtained higher sumrate compared to the without precoding scheme that is shown in Table 1 for interference considering scenario. We succeed to achieved maximum sumrate in MMSE for interference consideration environment and when we consider the idle scenario i.e., without interference, we got maximum sumrate without precoding that is discuss in Table 1 and Table 2, and Figure 2, Figure 3 and Figure 4 below. The pseudo-inverse of channel for ZF and MMSE are given with a weighting factor-

$$
\begin{gathered}
W_{Z F}=H^{H}\left(H H^{H}\right)^{-1} \\
W_{M M S E}=H^{H}\left(H H^{H}+\frac{\sigma^{2}}{\rho_{k}} I_{k}\right)^{-1}
\end{gathered}
$$

Where $\sigma$ is the AWGN, $I_{k}$ is the identity matrix of $G^{\text {th }}$ selected users and $\rho_{k}$ is the downlink transmit power. $\rho_{k}$ has to satisfy equation (5)

$$
\sum_{k \in X} \rho_{k} \leq \rho
$$

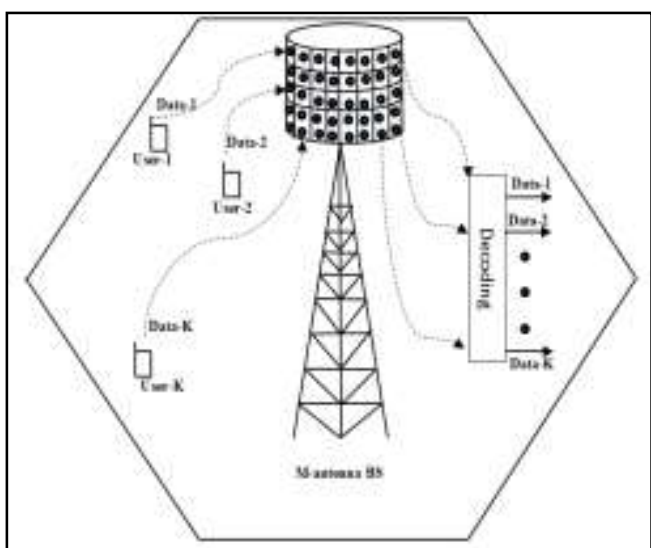

Figure-1(b): Uplink

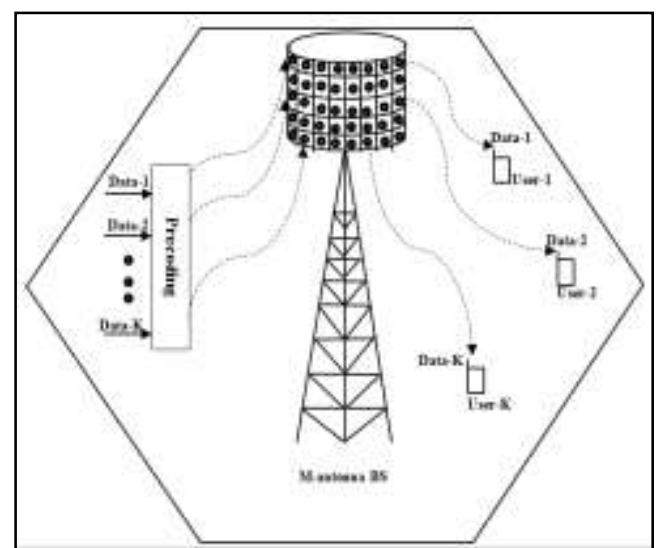

Figure-1(a): Downlink

Figure 1(a) and (b). Massive MIMO System with Combine User Selection and Antenna Scheduling

After applying the linear precoding the $k^{\text {th }}$ received signal vector can be rewritten as-

$$
\begin{aligned}
\tilde{y}_{Z F} & =W_{Z F} y_{k} \\
& =H_{k}{ }^{H}\left(H_{k} H_{k}{ }^{H}\right)^{-1} h_{k}^{H} s_{k}+W_{Z F} \sigma_{k}
\end{aligned}
$$




$$
\begin{aligned}
& \tilde{y}_{\text {MMSE }}=W_{\text {MMSE }} y_{k} \\
& \quad=H_{k}{ }^{H}\left(H_{k} H_{k}{ }^{H}+\frac{\sigma^{2}}{\rho_{d}} I_{k}\right)^{-1} h_{k}{ }_{k} s_{k}+W_{M M S E} \sigma_{k} .
\end{aligned}
$$

Where the first term in the right side of equation (6) and (7) is the pseudo-inverse of ZF and MMSE multiplies with desired signal and $k^{\text {th }}$ channel gain vector respectively. In addition, the second term is background AWGN multiplies with $W_{Z F}$ and $W_{M M S E}$ respectively. The uplink and downlink combined user selection and antenna scheduling massive MIMO system with precoding scenario is shown in Figure 1 (a) and (b) in above.

\subsection{Problem Formulation}

We consider $\rho$ as total transmit power and equally distributed to all users, an optimal power allocation water filling algorithm is used. The users to get equal power must follow equation (8).

$$
\rho_{k}=\left(\mu-\frac{1}{\left|h_{k}^{H} W_{k}\right|^{2}}\right)^{+}
$$

Follow equation (9) to choose the water level $\mu$,

$$
\sum_{m \in A} \sum_{k \in U} \rho_{k}=\rho
$$

As $\rho$ and $N$ are limited, so BS antenna can serve only $N$ users at the same time. We have considered $A_{S S A}$ and $U_{S O U}$ as selected number of antennas and users set respectively. The final vector of antenna selection and user scheduling and multiplication of pseudo-inverse of ZF and MMSE vector are given by-

$$
\begin{aligned}
& \tilde{y}_{k, \mathrm{ZF}}=h_{k}{ }^{H} w_{k, \mathrm{ZF}} s_{k}+\sum_{i \neq k, k \in U} h_{k}{ }^{H} w_{i, \mathrm{ZF}} s_{i}+\sigma_{k} \\
& \tilde{y}_{k, \mathrm{MMSE}}=h_{k}{ }^{H} w_{k, \mathrm{MMSE}} s_{k}+\sum_{i \neq k, k \in U} h_{k}{ }^{H} w_{i, \mathrm{MMSE}} s_{i}+\sigma_{k}
\end{aligned}
$$

The first term of the equation (10) and (11) is the desired signal vector; second and third terms are the interference and noise components respectively. The received signal to interference plus noise ratio (SINR) and signal to noise ratio (SNR) for selected users and scheduled antennas are given by equation (12) and (13) for ZF and (14) and (15) is for MMSE respectively,

$$
\begin{aligned}
& \operatorname{SINR}_{Z F}=\frac{\rho_{k}\left|h_{k}{ }^{H} w_{k, Z F}\right|^{2}}{\sum_{i \neq k, k \in U} \rho_{i}\left|h_{k}{ }^{H} w_{i, \mathrm{ZF}}\right|+\sigma_{k}} \\
& S N R_{Z F}=\rho_{k}\left|h_{k}{ }^{H} w_{k, Z F}\right|^{2} \\
& \operatorname{SINR}_{\text {MMSE }}=\frac{\rho_{k}\left|h_{k}{ }^{H} w_{k, M M S E}\right|^{2}}{\sum_{i \neq k, k \in U} \rho_{i}\left|h_{k}{ }^{H} w_{i, M M S E}\right|+\sigma_{k}} . \\
& S N R_{\text {MMSE }}=\rho_{k}\left|h_{k}{ }^{H} w_{k, M M S E}\right|^{2}
\end{aligned}
$$


The sumrate of selected users and scheduled antennas of broadcast massive MIMO systems are calculated using equation (16) and (17),

$$
\begin{aligned}
& R=\sum_{q=1}^{G} \log _{2}\left(1+S_{\left.I N R_{Z F / M M S E}\right)}\right) \\
& R=\sum_{q=1}^{G} \log _{2}\left(1+S N R_{Z F / M M S E}\right)
\end{aligned}
$$

\section{Two Proposed Algorithms and Description}

Numerous JASUS algorithms as mentioned in Section 1 which achieves higher system sumrate and resolve the computational complexity. However, maximum of those algorithms failed to obtain the goal of maximum system sumrate. In this section, we propose combined semi-orthogonal user selection and antenna scheduling algorithm to enhance system sumrate and lower the computational complexity. For user selection, we consider semi-orthogonal based user selection scheme. The advantage of this scheme in massive MIMO systems is that possibility of getting semi-orthogonal users among them is very high because of large number of users. The semi-orthogonal users are selected iteratively and store them by array in matrix. The remaining users are removed from the systems at the end of the user selection, which implies that it efficiently utilized power and spectrum. The algorithm stops when required number of users are selected. The steps of semi-orthogonal user selection are presented in algorithm-1. The combined users and antenna selection, and scheduling step discussed in algorithm-2. The algorithm-2 iteratively schedules those antennas with the selected users that have maximum channel gain.

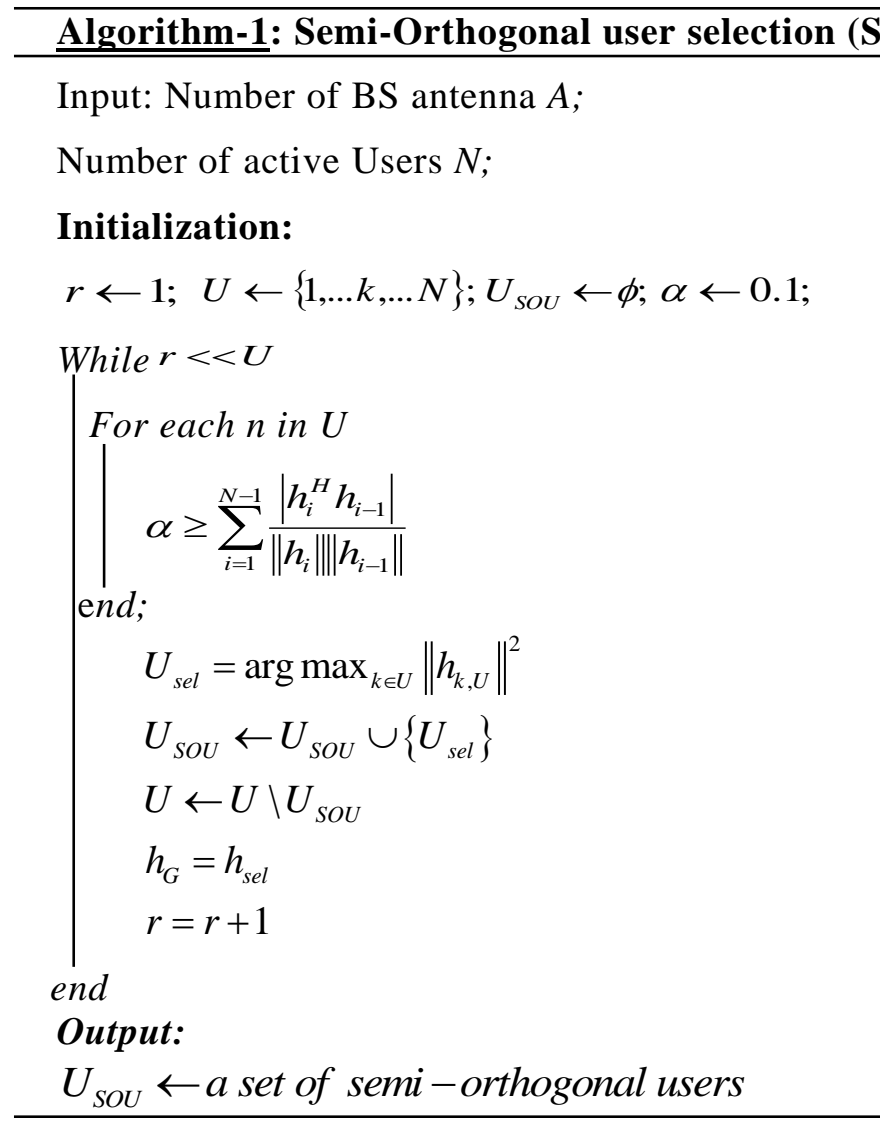




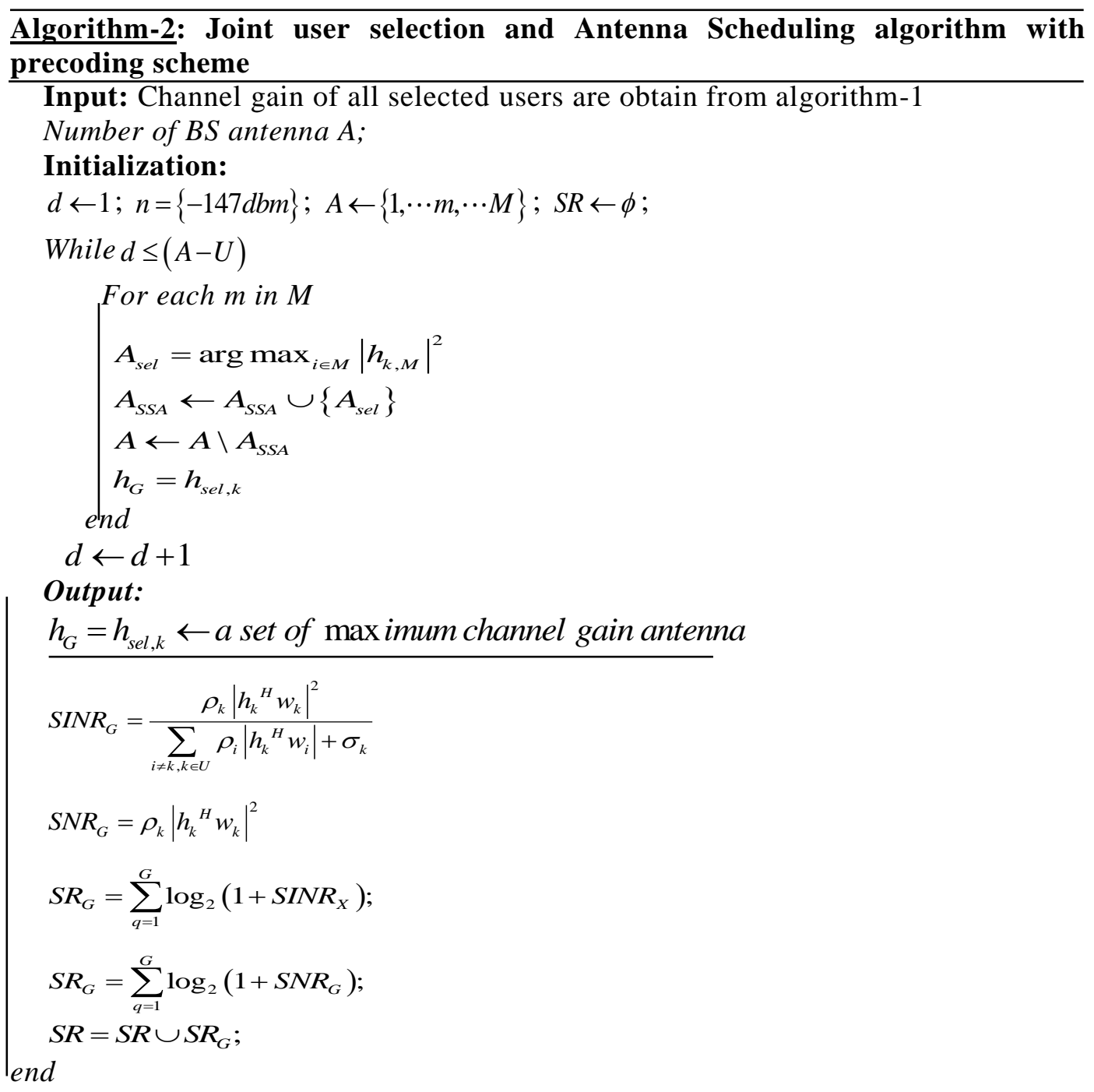

\section{Detail Analysis of Result}

Throughout this paper, we have investigated the performance of sumrate and computational complexity of the proposed algorithm. In our proposed work, we assume a massive MIMO system with a single cell containing $M=40 / 50 / 60$ number of $B S$ antennas and $\mathrm{N}=20 / 30 / 40$ number of users having a single receive antenna. Among these antennas and users, 8 users and antennas have been selected, and compare the sumrate and complexity for different precoding techniques like ZF, MMSE and without precoding.

In Table 1, we have shown the comparison of simulation results in terms of sumrate considering the interference for different precoding. In Table 2, the sumrate comparison for different precoding scheme without interference is shown. We vary the transmitted power and calculate the sumrate for all precoding techniques. We also vary the spatial diversity and multiuser diversity of the system, and select 8 users and antennas, and schedule them and calculated the systems sumrate. From Table 1 and Table 2, it is clear that when power and diversity of the BS antenna's and user's increases, the sumrate is also increased in this massive MIMO systems. Hence, we can say that our algorithm is comfortable for large dimension massive MIMO scenario. For computational complexity issues we limit the selected number of users and schedule antennas upto 8.

From the Table 1 and Table 2, and Figure 2, Figure 3 and Figure 4 it is concluded that for different transmit power and BS antennas of massive MIMO systems, the sumrate is enhanced at MMSE and lowest at without precoding when interference is considered. 
Moreover, sumrate is maximum when interference and precoding is not considered and lower when MMSE precoding is used. It is noticed that the sumrate is increased with the proposed algorithms compared to the TCB JASUS, JASUS, and greedy based JASUS [8] algorithm when increased the diversity as well as transmit power.

Table 1. Average sumrate of Combined Antenna and User Selection Algorithm. 8 BS Antennas and Same Number of users are Selected from $\mathrm{M}=40 / 50 / 60$ Active Antennas and $\mathrm{N}=20 / 30 / 40$ Active Users for Different Precoding Scheme

\begin{tabular}{|c|c|c|c|}
\hline Transmit power $(\mathrm{dB})$ & MMSE & $\mathrm{ZFBF}$ & Without Precoding \\
\hline & \multicolumn{3}{|c|}{$M=40 ; N=20$} \\
\hline $20 \mathrm{~dB}$ & 53.9089 & 51.7356 & 32.6777 \\
\hline 30dB & 76.2299 & 73.8616 & 57.8897 \\
\hline $35 \mathrm{~dB}$ & 98.8304 & 87.5111 & 67.9892 \\
\hline \multirow[t]{2}{*}{$40 \mathrm{~dB}$} & 105.8673 & 102.1534 & 84.1601 \\
\hline & \multicolumn{3}{|c|}{$M=50 ; N=30$} \\
\hline $20 \mathrm{~dB}$ & 54.6755 & 53.7652 & 33.5758 \\
\hline 30dB & 78.8269 & 75.6920 & 58.7600 \\
\hline $35 \mathrm{~dB}$ & 98.6885 & 93.8968 & 68.8557 \\
\hline \multirow[t]{2}{*}{$40 \mathrm{~dB}$} & 105.7418 & 100.0989 & 85.0253 \\
\hline & \multicolumn{3}{|c|}{$M=60 ; N=40$} \\
\hline $20 \mathrm{~dB}$ & 57.8889 & 52.0269 & 33.6429 \\
\hline 30dB & 81.0861 & 77.8591 & 59.1212 \\
\hline 35dB & 98.8476 & 95.0393 & 72.3129 \\
\hline $40 \mathrm{~dB}$ & 108.6029 & 103.7243 & 85.5698 \\
\hline
\end{tabular}

Table 2. Average sumrate from Combined Antenna and User Selection Algorithm. 8 BS Antennas and Same Number of Users are Selected from $M=40 / 50 / 60$ Active Antenna and $N=20 / 30 / 40$ Active Users without Interference Consideration for different Precoding Scheme

\begin{tabular}{|l|l|l|l|}
\hline Transmit Power (dB) & Without Precoding & \multicolumn{3}{|c|}{ ZFBF } & MMSE \\
\hline & \multicolumn{3}{|c|}{ M=40; N=20 } \\
\hline $\mathbf{2 0 d B}$ & 62.4761 & 35.8467 & 23.5362 \\
\hline $\mathbf{3 0 d B}$ & 88.8328 & 58.8914 & 52.2404 \\
\hline $\mathbf{3 5 d B}$ & 102.0335 & 70.6528 & 59.2700 \\
\hline $\mathbf{4 0 d B}$ & 115.2369 & 85.0342 & 78.3357 \\
\hline & \multicolumn{3}{|c|}{ M=50; N=30 } \\
\hline $\mathbf{2 0 d B}$ & 64.0556 & 35.9578 & 26.0157 \\
\hline $\mathbf{3 0 d B}$ & 90.4194 & 59.1673 & 53.9629 \\
\hline $\mathbf{3 5 d B}$ & 103.6206 & 71.0938 & 66.9539 \\
\hline $\mathbf{4 0 d B}$ & 116.8242 & 85.4902 & 80.1958 \\
\hline & & $\mathbf{M}=\mathbf{6 0} ; \mathbf{N}=\mathbf{4 0}$ & \\
\hline $\mathbf{2 0 d B}$ & 65.6453 & 36.5508 & 27.4690 \\
\hline $\mathbf{3 0 d B}$ & 92.1807 & 62.5230 & 54.8269 \\
\hline $\mathbf{3 5 d B}$ & 105.4653 & $\mathbf{7 2 . 6 5 0 2}$ & 68.6865 \\
\hline $\mathbf{4 0 d B}$ & 118.7521 & 86.5643 & 84.3689 \\
\hline
\end{tabular}




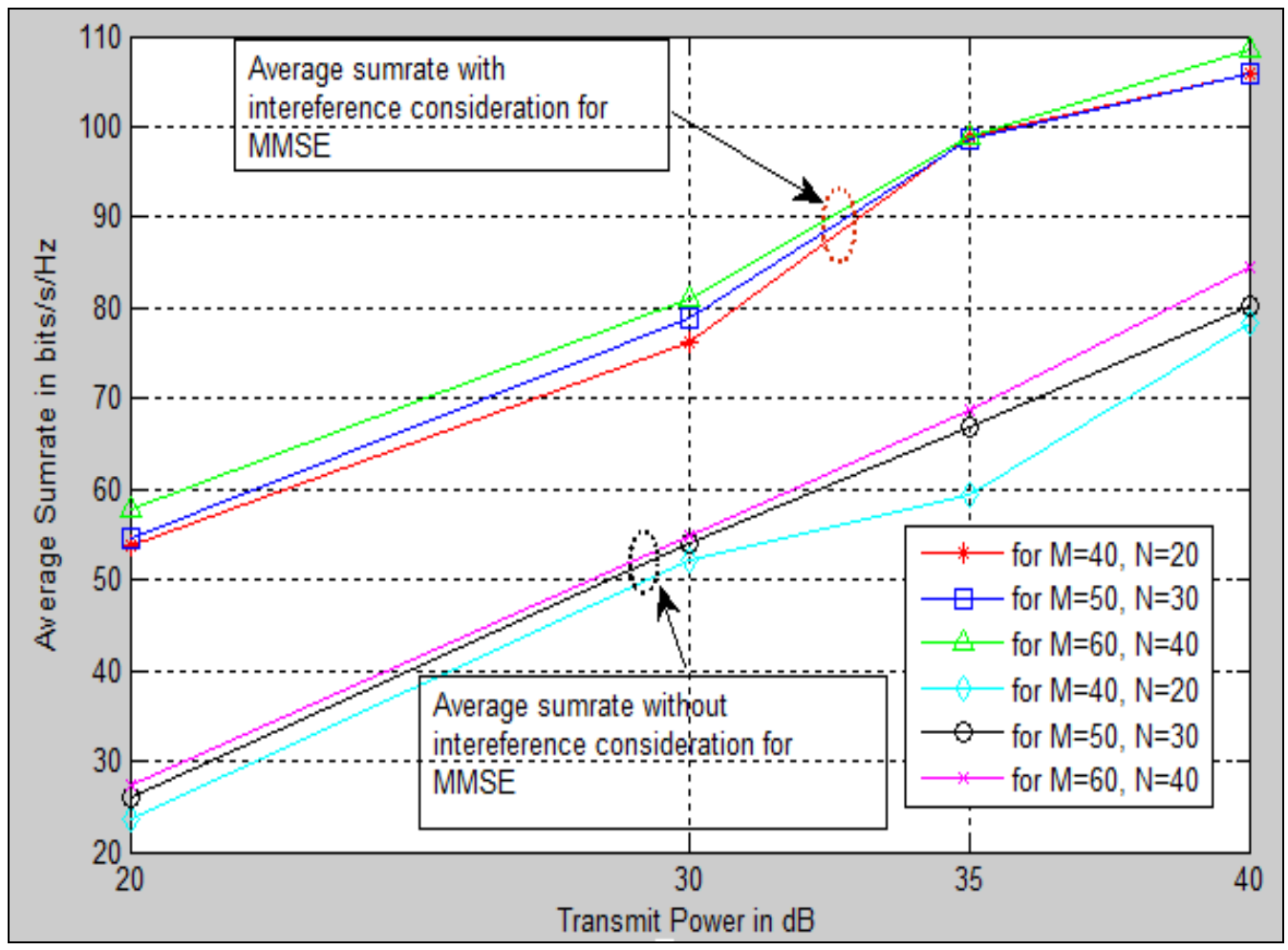

Figure 2. Average sumrate from combined Antenna Selection and User Scheduling Algorithm with and without Interference Consideration for MMSE

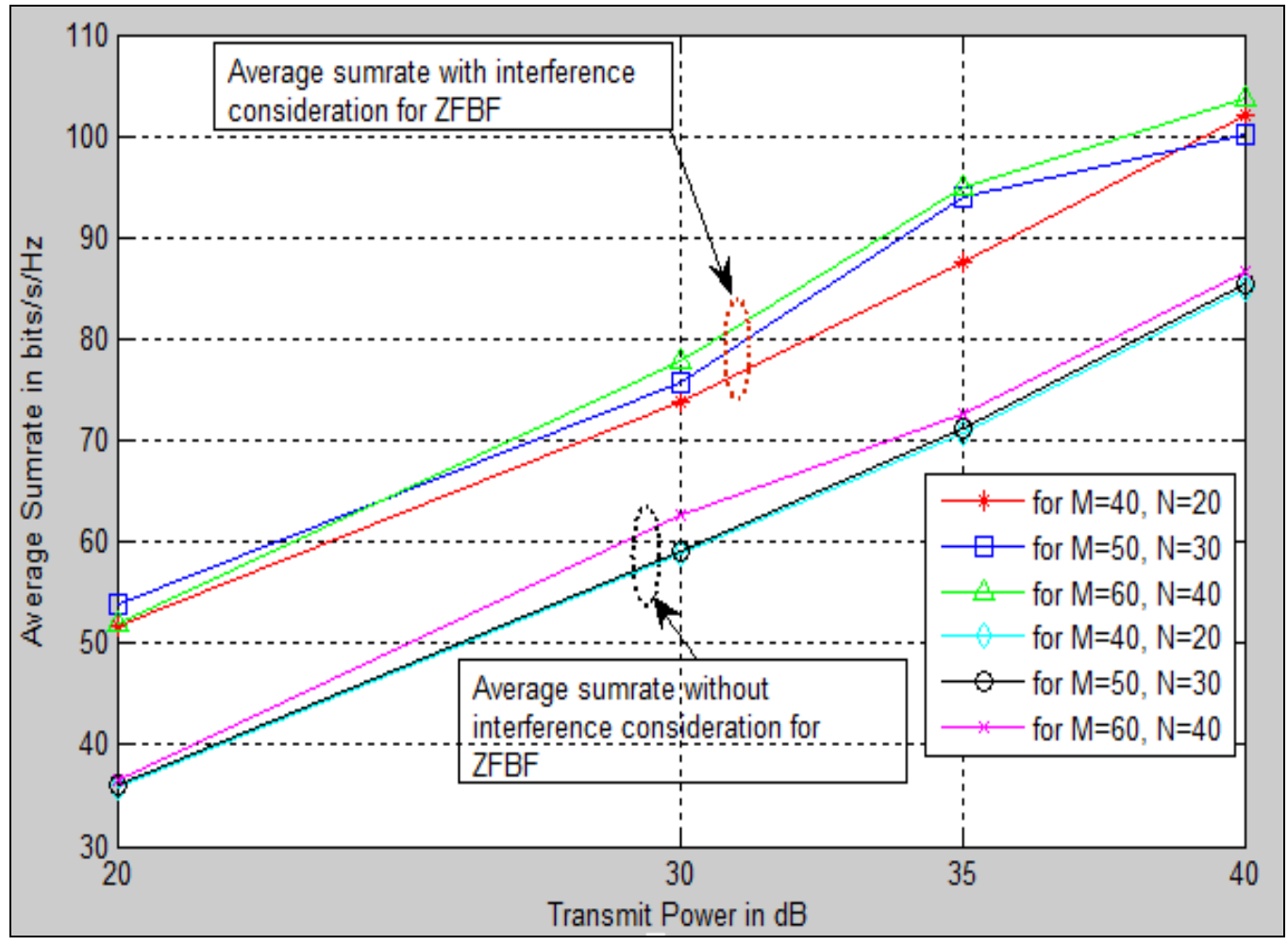

Figure 3. Average sumrate of combine Antenna Selection and User Scheduling with and without Interference Consideration for ZFBF 


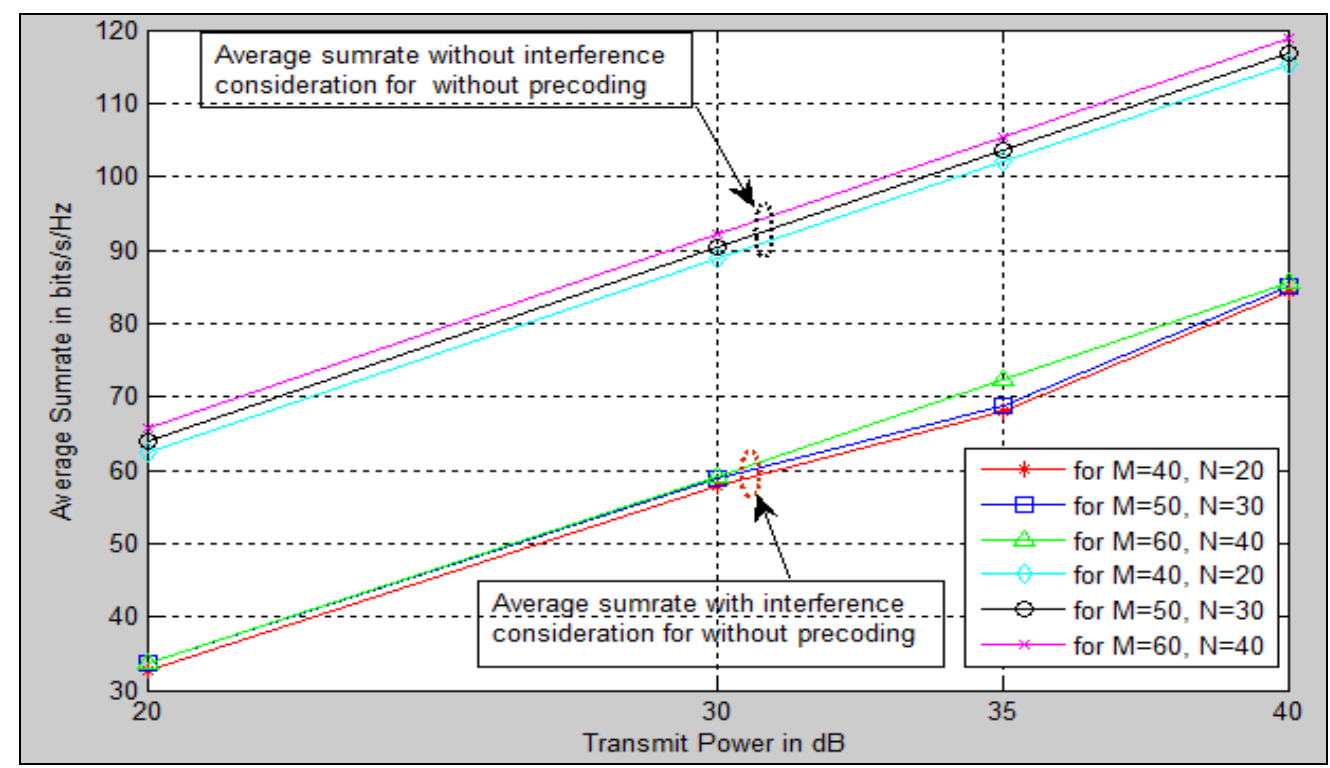

Figure 4. Average sumrate using Combined Antenna Selection and User Scheduling Algorithm with and without Interference Consideration when Precoding is not used

\section{Conclusion}

In this paper, we have explored how to improve the system sumrate in massive MIMO systems by employing joint user selection and antenna scheduling algorithm. In our proposed works, algorithm- 1 has been used to select the users which are semi-orthogonal to each other and removed the remaining users from the systems. In algorithm-2, we select and schedule the antennas that have maximum channel gain with the already selected users employing algorithm-1 to obtain maximum system sumrate. In our algorithms we have calculated the sumrate for two environments, firstly, by considering the interference and secondly for without considering the interference and compared them. Moreover, our results show that the sumrate is enhanced compared to the other proposed works that is obtained in [5] and [7-9].

\section{Acknowledgments}

The author thanks Ministry of electronics and Information Technology (Meity), Government of India, for financial support to carry out this work.

\section{References}

[1] E. G. Larsson, F. Tufvesson, O. Edfors and T. L. Marzetta, "Massive MIMO for next generation wireless systems", IEEE Commun. Mag., vol. 52, no. 2, (2014), pp. 186-195.

[2] H. Quoc Ngo, "Massive MIMO: Fundamentals and System Designs", Linköping Studies in Science and Technology, Dissertations, No. 1642, Linköping University, SE-581 83 Linköping, Sweden, (2015).

[3] T. L. Marzetta, E. G. Larsson, H. Yang and H. Q. Ngo, "Fundamentals of Massive MIMO", Cambridge, UK: Cambridge University Press, (2016).

[4] M. Jo, D. Araujo, T. Maksymyuk, A. Almeida and T. F. Maciel, "Massive MIMO: Survey and Future Research Topics", IET Commun., (2016), pp. 1-26.

[5] Y. Dong, Y. Tang and K. Zhang, "Improved Joint Antenna Selection and User Scheduling for Massive MIMO Systems", ICIS 2017, Wuhan, China, (2017), pp. 69-74.

[6] Y. Gao, W. Jiang and T. Kaiser, "Bidirectional branch and bound based antenna selection in massive MIMO systems", Proc. IEEE 26th PIMRC, Hong Kong, (2015), pp. 563-568.

[7] M. Benmimoune, E. Driouch, W. Ajib and D. Massicotte, "Joint transmit antenna selection and user scheduling for Massive MIMO systems", IEEE Wireless Communications and Networking Conference (WCNC), New Orleans, LA, (2015), pp. 381-386. 
[8] G. Xu, A. Liu, W. Jiang, H. Xiang and W. Luo, "Joint user scheduling and antenna selection in distributed massive MIMO systems with limited backhaul capacity", China Communications, vol. 11, no. 5, (2014), pp. 17-30.

[9] X. Liu and X. Wang, "Efficient Antenna Selection and User Scheduling in 5G Massive MIMO-NOMA System”, IEEE 83rd Vehicular Technology Conference (VTC Spring), Nanjing, (2016), pp. 1-5.

[10] A. Tomasoni, G. Caire, M. Ferrari and S. Bellini, "On the selection of semi-orthogonal users for zeroforcing beamforming”, IEEE International Symposium on Inform Theory (ISIT), (2009), pp. 1100-1104.

[11] B. Lee, L. Ngo and B. Shim, "Antenna group selection based user scheduling for massive MIMO systems”, IEEE Global Communications Conference, Austin, TX, (2014), pp. 3302-3307.

[12] N. Jindal and A. Goldsmith, "Dirty-paper coding versus TDMA for MIMO broadcast channels", IEEE Trans. Inf. Theory, vol. 51, no. 5, (2005), pp. 1783-1794.

\section{Authors}

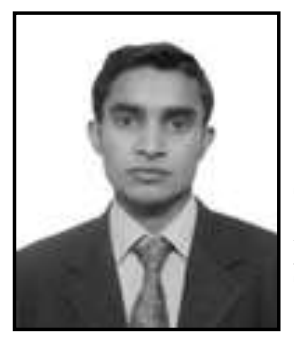

Tasher Ali Sheikh, received B.Tech. degree in Electronics and Communication Engineering from Central Institute of Technology (CIT), Kokrajhar, Assam, India, in 2013, M.Tech. in Mobile Communication and Computing from National Institute of Technology (NIT), Arunachal Pradesh, India, in 2015, and currently pursuing Ph.D. from North Eastern Regional Institute of Science and Technology (NERIST), Arunachal Pradesh, India. He is an IEEE Student Member and Lifetime member of International Association of Engineers (IAENG), Member of American Association of Science and Technology (AASCIT). His field of interest includes Massive MIMO, Microwave communication and wireless communication.

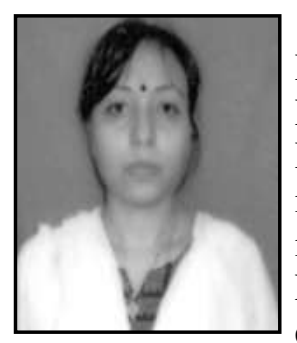

Joyatri Bora, is currently working as an Assistant Professor in the Department of ECE at NERIST Deemed University, India. She did $\mathrm{PhD}$ in Electronics \& Communication Engineering from NERIST Deemed University, India in 2015. She received her M. Tech degree in Electronics Design and Technology from Tezpur University, India in 2002 and B. Tech degree in Electrical Engineering from Jorhat Engineering College, India in 1995. She has more than 10 years of experience in research and teaching field. She has published research articles in International referred journals and conferences. Her research interest includes Wireless Communication, Massive MIMO, and Bioelectronics.

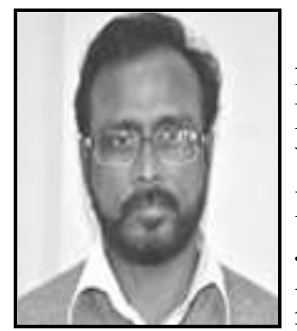

Md. Anwar Hussain, received B.Sc. degree in Physics honours from Gauhati University, Assam, India, in 1981, B.Tech. and M.Tech. in Radio Physics \& Electronics from Calcutta University, West Bengal, India, in 1985 and 1987 respectively. Also he received $\mathrm{PhD}$ degree in Electronics and Communication Engineering from Jadavpur University, West Bengal, India, in 2002. He is currently a professor in the department of Electronics and Communication Engineering at North Eastern Regional Institute of Science and Technology (NERIST), Arunachal Pradesh, India. He has more than 31 years of experience in research and teaching field. His research interests includes massive MIMO, High data rate wireless communication \& networks, Routing \& scheduling in Multi-hop wireless networks, Key distribution in Sensor networks, Mobile computing security, Time-series data modeling and prediction, Networks-on-Chip. 